EGU2020-13609, updated on 10 Jun 2021

https://doi.org/10.5194/egusphere-egu2020-13609

EGU General Assembly 2020

(c) Author(s) 2021. This work is distributed under

the Creative Commons Attribution 4.0 License.

\title{
Soil carbon respiration in tropical forest soils along geomorphic and geochemical gradients
}

\author{
Benjamin Bukombe ${ }^{1}$, Laurent Kidinda ${ }^{2}$, Alison Hoyt ${ }^{3}$, Cordula Vogel ${ }^{2}$, Marijn Bauters ${ }^{4}$, Florian \\ Wilken ${ }^{1}$, Karsten Kalbitz ${ }^{2}$, Peter Fiener ${ }^{1}$, and Sebastian Doetterl ${ }^{1,5}$ \\ ${ }^{1}$ Institute of geography, University of Augsburg, Augsburg, Germany \\ ${ }^{2}$ Institute of soil science and site ecology, Dresden University of Technology, Dresden, Germany \\ ${ }^{3}$ Biogeochemistry, Max Plank Institute Jena, Jena, Germany \\ ${ }^{4}$ ISOFYS, Gent University, Ghent, Belgium \\ ${ }^{5}$ Terrestrial Ecosystems, ETH Zürich, Zürich, Switzerland.
}

Tropical ecosystems and the soils therein have been reported as one of the most important and largest terrestrial carbon (C) pools and are considered important climate regulator. Carbon stabilization mechanisms in these ecosystems are often complex, as these mechanisms crucially rely on the interplay of geology, topography, climate, and biology. Future predictions of the perturbation of the soil carbon pool ultimately depend on our mechanistic understanding of these complex interactions.

Using laboratory incubation experiments, we investigated if carbon release from soils through heterotrophic respiration in the African highland forests of the Eastern Congo Basin follows predictable patterns related to topography, soil depth or geochemical soil properties that can be described at the landscape scale and ultimately be used to improve the spatial accuracy of soil $C$ respiration in mechanistic models. In general, soils developed on basalt and granite parent material (mafic and felsic geochemistry of parent material) showed significantly $(p<0.05)$ higher specific respiration than soils developed on sedimentary rocks (mixed geochemistry) with highest rates measured for soils developed on granite. For soils developed on basalt, specific respiration decreased two-fold with soil depth, but not for soils developed on granite or sedimentary rocks. No significant differences in respiration under tropical forest were found in relation to topography for any soil and geochemical background.

Using a non-linear, stochastic gradient boosting machine learning approach we show that soil biological, physical and chemical properties can predict the pattern of specific soil respiration $\left(R^{2}=0.41, p<0.05\right)$. An assessment of the relative importance of the included predictors for soil respiration resulted in $43 \%$ of the model being driven by geochemistry (pedogenic oxides, nutrient availability), $12 \%$ driven by soil texture and clay mineralogy, $34 \%$ by microbial biomass, $\mathrm{C}: \mathrm{N}$, and $\mathrm{C}: \mathrm{P}$ ratios and $11 \%$ by topographic indices.

We conclude that, in order to explain soil $\mathrm{C}$ respiration patterns in tropical forests, a complex set of variables need to be considered that differs depending on the local bedrock chemistry. Its effect 
is likely related to the varying strength of $C$ stabilization with minerals as well as nutrient availability that might drive $C$ input patterns and microbial turnover. 